\title{
Patient-reported outcomes: association between physical activity and quality of life in patients with chronic kidney disease
}

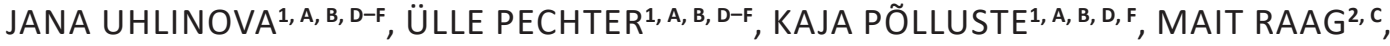 \\ ORCID ID: 0000-0002-3778-6815 \\ RAILI MÜLLER ${ }^{1, A, B, D}$, RIINA KALLIKORM ${ }^{1, A, D}$, MARGUS LEMBER ${ }^{1, A, ~ F, ~ G, ~ M A I ~ O T S-R O S E N B E R G ~}{ }^{1, A, ~ B, ~ D-F ~}$ \\ ${ }^{1}$ Department of Internal Medicine, University of Tartu and Tartu University Hospital, Tartu, Estonia \\ ${ }^{2}$ Department of Epidemiology and Mathematics, University of Tartu, Tartu, Estonia
}

A - Study Design, B - Data Collection, C - Statistical Analysis, D - Data Interpretation, E - Manuscript Preparation, F - Literature Search, G - Funds Collection

Summary Background. Lifestyle factors contribute to the risk of progression of chronic kidney disease (CKD). CKD patients are getting older, have decreased physical activity (PA) and increased psychosocial problems. Muscle weakness and fatigue causes poor functional ability, especially with impaired renal function.

Objectives. The aim of the study was to find associations between PA and self-reported patients' outcomes in CKD patients.

Material and methods. Consecutive adult patients with CKD (total $n=130$; estimated glomerular filtration rate (eGFR) $\geq 45 \mathrm{~mL}$ $/ \mathrm{min} / 1.73 \mathrm{~m}^{2} n=54$; eGFR $<45 \mathrm{~mL} / \mathrm{min} / 1.73 \mathrm{~m}^{2} n=76$ ) or other chronic conditions $(n=247)$ referred to Internal Medicine Clinic were recruited. A population-based control group was randomly selected ( $n=327)$. The International Physical Activity Questionnaire (IPAQ) short form was opted to assess PA. The 36-Item Short Form Health Survey (SF-36) was used to assess patients' health-related quality of life (HRQOL).

Results. All HRQoL dimension scores in patients with noticeably reduced kidney function (eGFR $<45 \mathrm{ml} / \mathrm{min} / 1.73 \mathrm{~m}{ }^{2}$ ) were statistically significantly lower in comparison with other groups, except the mental health (RE) score. In the CKD low group, the PA level significantly influenced both overall physical component score (PCS) and subscales and was strongly related with walking habits (PCS, $p=0.0002$ ). Walking positively influenced the mental component score (MCS, $p=0.007$ ) in the CKD low group.

Conclusions. We found that subjects with severely impaired kidney function and good walking habits have higher quality of life scores. Using these instruments for measurement, we can evaluate patient-reported outcomes, and regular control helps us to improve quality of life among patients with CKD.

Key words: quality of life, exercise, renal insufficiency, chronic.

Uhlinova J, Pechter Ü, Põlluste K, Raag M, Müller R, Kallikorm R, Lember M, Ots-Rosenberg M. Patient-reported outcomes: association between physical activity and quality of life in patients with chronic kidney disease. Fam Med Prim Care Rev 2020; 22(4): 343-348, doi: https://doi.org/10.5114/fmpcr.2020.100448.

\section{Background}

Chronic kidney disease (CKD) is a serious health problem throughout the world and is one of the most potent known risk factors for cardiovascular disease. There is increasing evidence that lifestyle factors have an impact on the risk of progression of CKD. CKD patients are getting older, are almost invariably hypertensive and have decreased physical activity (PA) and increased psychosocial problems [1]. Muscle weakness and fatigue causes poor functional ability, especially when renal dysfunction becomes aggravated [2]. PA is associated with the stage of CKD [3]. If patients choose to smoke or use alcohol excessively, the additive risk is profound [4-6]. The risk of atherosclerotic events in patients with CKD, especially in patients on renal replacement therapy, has shown to be 10-20 times greater than in the general population [7-9]. Thus, these statements reflect the importance of ensuring lifestyle recommendations. According to the recommendations of Kidney Disease Improving Global Outcomes (KDIGO), people with CKD should be encouraged to undertake PA compatible with cardiovascular health and tolerance, achieve a healthy weight and stop smoking [10]. Exercise [11], weight loss [12] and smoking cessation [4] in CKD patients are equally important in all countries, and thus this recommen- dation is of international relevance and applicability. KDIGO recommendations stress the importance of preventive measures in renal patients as early as possible in the course of kidney failure, when these can be most effective, cost efficient and of the greatest benefit to patients and society. Currently, two well-conducted meta-analyses have shown the benefits of low-intensity physical activity in patients with CKD $[13,14]$. A multiple-riskfactor intervention is suggested, including PA, a special care plan for nutritional management and lifestyle counselling based on patients' medical and social conditions [15-17]. The results of a recent systematic review by MacKinnon and others showed an association of self-reported physical function with survival in patients with CKD [18]. Therefore, various insights into the qualities and consequences of the everyday life of patients living with kidney disease are needed [19].

\section{Objectives}

We hypothesised that PA improves quality of life in patients with chronic kidney disease independently of CKD stage. The aim of the study was to find associations between PA and self-reported patient outcomes in CKD patients. The second aim of 
the study was to compare the mean data of CKD patient groups with a group with other chronic conditions (CC) and with control subjects.

\section{Material and methods}

\section{Data sources}

In this cross-sectional study, with a duration of 6 months, 758 consecutive adult patients (aged $>18$ ) visiting the outpatient Internal Medicine Clinic at Tartu University Hospital and 6 primary health care centres on randomly selected days were included in the study. For the current study, the data was collected under two large institutional projects [20]. Exclusion criteria was being under 18 years of age and refusal to participate in study. Final data analysis consisted of 704 subjects, as 54 questionnaires were not returned or were not properly filled out. According to the KDIGO guidelines, 130 patients had been diagnosed with CKD, and CKD was defined by eGFR and albuminuria. The CKD group was made up of $21.5 \%$ diabetic and $17.6 \%$ hypertensive patients. The patients with CKD were divided into two groups - patients with estimated glomerular filtration rate (eGFR) $<45$ $\mathrm{mL} / \mathrm{min} / 1.73 \mathrm{~m}^{2}$ and eGFR $\geq 45 \mathrm{~mL} / \mathrm{min} / 1.73 \mathrm{~m}^{2}$. This divide was used because of the different outcomes and risk profiles in these CKD patient groups [10]. The group with CC consisted of 247 patients with a diagnosis of one or more CC with no proteinuria or impaired kidney function. The most prevalent were disorders of the endocrine organs (including diabetes types 1 and 2), cardiovascular system (e.g. hypertension, heart failure, etc.) and musculoskeletal system (e.g. osteoarthritis, chronic low back pain, early rheumatoid arthritis, etc.). A population-based control group was recruited from Primary Health Care ambulatory office visits and consisted of 327 subjects. Participation was voluntary and anonymous. A signed written informed consent was obtained from the participants. The Ethics Review Committee on Human Research at the University of Tartu approved the study. All study patients were asked to fill out the paper questionnaires about PA, HRQoL and smoking history in the office waiting room or at home. BMI was calculated from the patient's individual body weight and height measured by a physician or nurse. According to World Health Organization criteria, normal weight was defined as $\mathrm{BMI}<25 \mathrm{~kg} / \mathrm{m}^{2}$, overweight as $\mathrm{BMI} 25-$ $-29.9 \mathrm{~kg} / \mathrm{m}^{2}$ and obesity as $\mathrm{BMI} \geq 30 \mathrm{~kg} / \mathrm{m}^{2}$.

\section{Physical activity}

The International Physical Activity Questionnaire (IPAQ) short form was used to assess PA because of its relatively good reliability and validity [21]. Daily life activities are known as the tasks of everyday life that are normal and independently performed, including self-care activities, walking, gardening, homemaking and other activities [22]. The IPAQ short version estimates how much health enhancing PA, including daily life activities and exercise, the person has undertaken over the previous seven days, divided into three intensities (vigorous PA, moderate PA and walking). We calculated the total PA (metabol- ic unit per week, MET min/week) as suggested in the Guidelines for Data Processing and Analysis of the IPAQ.

\section{Health-related quality of life}

The patients' health-related quality of life (HRQoL) was measured by the SF-36 [21, 23]. This instrument includes eight sub-scales - physical functioning (PF), role limitations due to physical problems (RP), bodily pain (BP), general health (GH), vitality (VT), social functioning (SF), role limitations due to emotional problems (RE) and mental health $(\mathrm{MH})$ - which allow for the calculation of physical component scores (PCS) and mental component scores (MCS), according to Wang and others [24]. The scale scores range from 0 to 100 , with higher scores indicating a better health status.

\section{Data analysis}

CKD patients were divided into two groups - CKD low (58.5\%) - patients with eGFR $<45 \mathrm{~mL} / \mathrm{min} / 1.73 \mathrm{~m}^{2}$, and CKD high (41.5\%) - with eGFR $\geq 45 \mathrm{~mL} / \mathrm{min} / 1.73 \mathrm{~m}^{2}$. BMI was categorised as $<18.5 \mathrm{~kg} / \mathrm{m}^{2}$ being underweight, $18.5-24.9 \mathrm{~kg} / \mathrm{m}^{2}$ being normal weight, $25.0-29.9 \mathrm{~kg} / \mathrm{m}^{2}$ being overweight and $\geq 30 \mathrm{~kg} / \mathrm{m}^{2}$ being obese. Based on total MET score, the subjects were grouped as low PA (score < $600 \mathrm{MET}$ min/week), moderate PA (score 600-3,000 MET min/week) and high PA (score $>3,000 \mathrm{MET} \mathrm{min} /$ week). Distributions of gender, education and smoking among CC, CKD-low, CKD - high and control group subjects were compared by the chi-squared test, and distributions of age and BMI were compared by the Kruskal-Wallis test. Unadjusted medians and medians adjusted for age, gender, education and BMI were compared by quantile regression. Dimensions of HRQoL were compared by the Kruskal-Wallis test and by linear regression adjusted for age, gender, education, BMI and smoking. Associations between risk factors and PA scores were analysed by quantile regression (unadjusted and adjusted for age, gender, education, BMI and smoking). Associations between $\mathrm{HRQOL}$ scores and risk factors were analysed by linear regression (unadjusted and adjusted for age, gender, education, $\mathrm{BMI}$ and smoking). Associations between HRQoL and PA scores were analysed by the Spearman rank-correlation coefficient and quantile regression adjusted for age, gender, education, BMI and smoking. $P$-values were adjusted by the Holm-Bonferroni correction for multiple testing and were compared with a significance level of 0.05. Data analysis was performed in $R \vee 3.2 .1$ with quantreg and ggplot2 packages.

\section{Results}

\section{Baseline characteristics of the study patients}

Table 1 illustrates the background characteristics of all study patient groups. There are more men in CC group $(p=0.05)$. The control group is younger $(p<0.0001)$. Education is lower in the CKD low group $(p=0.03)$. BMI is lower in the control group $(p<0.0001)$.

\begin{tabular}{|l|l|l|l|l|l|l|}
\hline Table 1. Characteristics of patients & \multicolumn{1}{l|}{} \\
\hline Parameter & & CC $(n=247)$ & CKD low $(n=76)$ & CKD high $(n=54)$ & Control $(n=327)$ & Total $(n=704)$ \\
\hline Gender & F & $65.2 \%$ & $56.6 \%^{\#}$ & $55.6 \%^{\#}$ & $53.8 \%$ & $58.3 \%$ \\
& M & $34.8 \%$ & $43.4 \%^{\#}$ & $44.4 \%^{\#}$ & $46.2 \%$ & $41.7 \%$ \\
\hline Age & Mean & $57.1(12.4)$ & $60.8(14.0)^{*}$ & $56.4(15.0)^{*}$ & $47.8(16.4)$ & $53.2(15.6)$ \\
\hline Education & Basic & $8.5 \%$ & $17.1 \%^{* \#}$ & $5.6 \%$ & $5.2 \%$ & $7.7 \%$ \\
& Upper sec. & $48.2 \%$ & $47.4 \%$ & $48.1 \%$ & $47.7 \%$ & $47.9 \%$ \\
& Tertiary & $43.3 \%$ & $35.5 \%^{* \#}$ & $46.3 \%$ & $47.1 \%$ & $44.4 \%$ \\
\hline BMI & Mean & $31.7(6.8)$ & $29.1(6.7)$ & $30.4(7.8)$ & $27.3(5.3)$ & $29.2(6.5)$ \\
\hline BMI class & Underweight & $0.0 \%$ & $3.2 \%^{* \#}$ & $5.4 \%^{* \#}$ & $1.5 \%$ & $1.4 \%$ \\
& Normal & $15.2 \%$ & $29.0 \%^{* \#}$ & $18.9 \%^{* \#}$ & $36.7 \%$ & $27.3 \%$ \\
& Overweight & $27.8 \%$ & $32.3 \%$ & $27.0 \%$ & $32.1 \%$ & $30.3 \%$ \\
& Obesity & $57.0 \%$ & $35.5 \%^{* \#}$ & $48.6 \%^{* \#}$ & $29.7 \%$ & $41.1 \%$ \\
\hline
\end{tabular}




\begin{tabular}{|l|l|l|l|l|l|l|}
\hline \multicolumn{2}{|l|}{ Table 1. Characteristics of patients } \\
\hline Parameter & & CC $(n=247)$ & CKD low $(n=76)$ & CKD high $(n=54)$ & Control $(n=327)$ & Total $(n=704)$ \\
\hline Smoking & Never & $61.0 \%$ & $86.8 \%^{* \#}$ & $90.7 \%^{* \#}$ & $52.9 \%$ & $62.4 \%$ \\
& Former & $18.7 \%$ & $6.6 \%^{* \#}$ & $1.9 \%^{* \#}$ & $26.6 \%$ & $19.7 \%$ \\
& Current & $20.3 \%$ & $6.6 \%^{* \#}$ & $7.4 \%^{* \#}$ & $20.5 \%$ & $17.9 \%$ \\
\hline
\end{tabular}

CC - chronic conditions, CKD - chronic kidney disease, BMI - body mass index, CKD low - eGFR < $45 \mathrm{ml} / \mathrm{min} / 1.73 \mathrm{~m}^{2}, \mathrm{CKD}$ high - eGFR ${ }^{3} 45 \mathrm{ml} /$ $/ \mathrm{min} / 1.73 \mathrm{~m}^{2}$; * statistically significant $p$-value $\leq 0.05$ compared to control group; " statistically significant $p$-value $\leq 0.05$ compared to CC group.
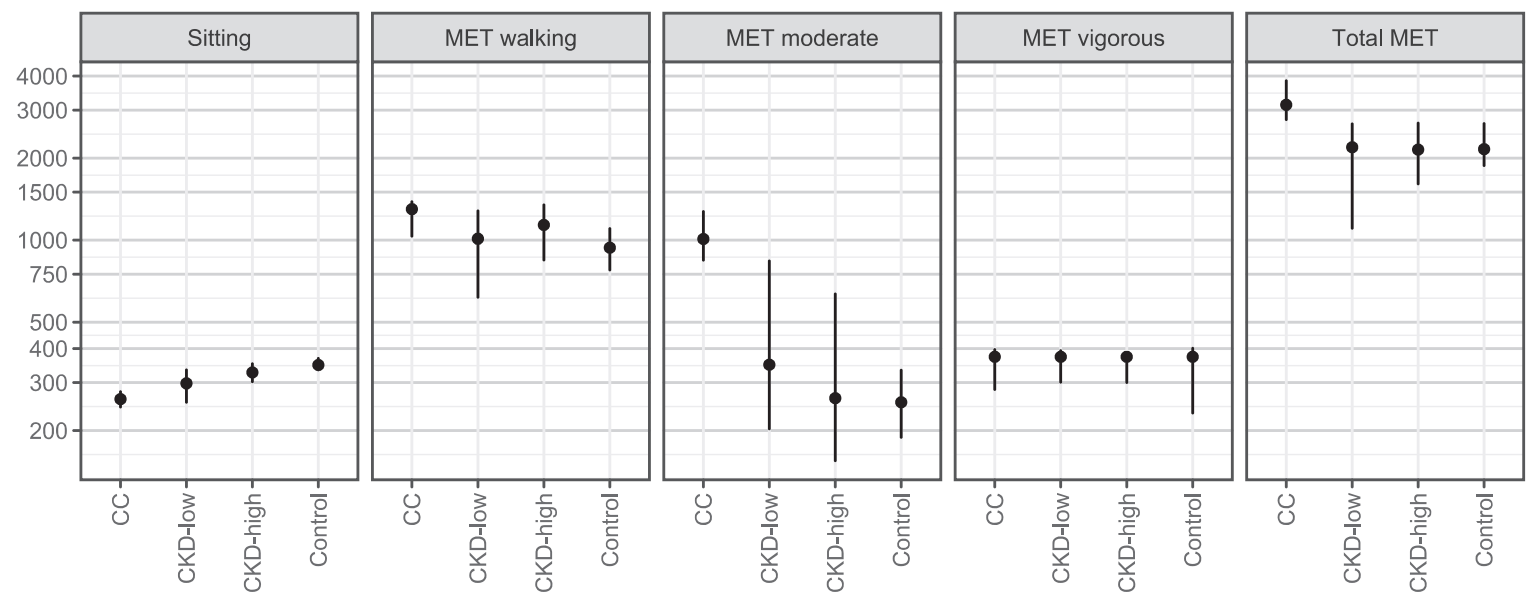

Figure 1. Median values of sitting time, MET (walking, moderate, vigorous) and total scores with $95 \%$ confidence intervals

CC - chronic condition, CKD - chronic kidney disease.

\section{Physical activity}

The distributions of measures of PA are given in Figure 1. According to the IPAQ analysis, the median value of total PA of CKD patients was significantly lower than CC patients. Most of the CKD patients reported moderate PA activity on past week and had a PA score between 600-3000 MET min/week. In comparison with the study groups, we found that the PA (total MET) of CKD patients was significantly lower than in CC patients $(p=$ 0.001). The PA of CKD patients of lower and higher eGFR did not differ significantly. Sitting more than six hours per day was reported by $28.5 \%$ of the patients. The average time spent sitting per day in minutes was $396.6 \pm 139.9$ (median 300). KruskalWallis multiple testing shows a significance in sitting time $(p<$ $0.001)$, walking $(p=0.05)$ and total PA $(p<0.0001)$. The results of Wilcoxon rank test adjusted for gender, age, education, BMI and smoking were as follows: CKD low vs CC group $(p=0.001)$, CKD high vs CC group ( $p=0.012)$ and CC vs control group $(p=0.012)$.

\section{BMI}

Mean BMI for the study group was $29.2 \pm 6.5 \mathrm{~kg} / \mathrm{m}^{2}$. Among all CKD patients, $71 \%$ were obese or overweight, and $4 \%$ were underweight. In the CC group, $84.8 \%$ of the patients were obese or overweight. The amount of obese or overweight subjects in the control group was $61.8 \%$. The BMI difference between the control group and other groups was significant $(p \geq 0.0001)$.

\section{Health-related quality of life}

The HRQoL scores in all dimensions (except RE) were significantly lower in the CKD low group in comparison with other groups. The physical score of the CKD high group was similar with the CC group, and the mental scores in the CKD group were high, similar to the CC and control groups. The scores of PCS and MCS were statistically significantly lower in CKD low group in comparing with other groups. The CKD high group PCS score was similar to the $\mathrm{CC}$ group score and lower than in the control group. MCS was not different in the CKD high, CC and control groups. Associations between HRQoL and lifestyle-related factors are presented in Table 2. SF-36 physical scores are associated with risk factors. In all study groups, PCS is significantly associated with age, gender, education, BMI and smoking, and MCS is strongly associated with age, gender and education.

The associations between HRQoL and PA in patients with CKD are presented in Table 3. Walking had a significant impact on PCS in the CKD low patient group. Total PA had a significant impact on the physical scores of SF-36 in the CKD low patient group.

\begin{tabular}{|l|l|l|l|l|l|}
\hline \multicolumn{6}{|l|}{ Table 2. Associations between HRQoL and lifestyle-related factors (physical and mental scores) } \\
\hline Risk factor & Physical score & PF & RP & BP & GH \\
\hline Age & a (a) & a (a) & a (a) & a (a) & a (a) \\
$20-42$ & $76.4(72.9-79.8)$ & $89.5(86.0-92.9)$ & $80.7(74.7-86.7)$ & $74.0(69.9-78.1)$ & $60.8(58.0-63.7)$ \\
$43-55$ & $64.1(60.5-67.6)$ & $77.0(73.4-80.5)$ & $64.1(57.9-70.2)$ & $63.6(59.4-67.8)$ & $51.7(48.7-54.6)$ \\
$56-65$ & $58.0(54.5-61.5)$ & $70.2(66.6-73.7)$ & $58.0(51.9-64.1)$ & $57.1(52.9-61.3)$ & $46.9(43.9-49.8)$ \\
$66-90$ & $48.6(45.0-52.2)$ & $56.5(52.9-60.1)$ & $42.0(35.8-48.2)$ & $54.7(50.5-59.0)$ & $40.5(37.5-43.4)$ \\
\hline Gender & a (0.0035) & a (a) & $0.0004(0.0438)$ & $0.0007(0.0134)$ & $0.0339(0.5070)$ \\
F & $58.4(55.9-60.8)$ & $68.5(65.9-71.0)$ & $56.6(52.4-60.8)$ & $59.3(56.5-62.2)$ & $48.7(46.6-50.7)$ \\
M & $67.0(64.1-69.9)$ & $80.6(77.6-83.6)$ & $68.3(63.3-73.2)$ & $66.9(63.6-70.2)$ & $52.1(49.7-54.5)$ \\
\hline Education & a (0.0028) & a (0.0097) & $0.0057(0.2123)$ & a (0.0017) & a (0.0030) \\
basic & $50.1(43.5-56.7)$ & $60.6(53.6-67.5)$ & $49.5(38.2-60.9)$ & $49.9(42.3-57.5)$ & $40.4(34.9-45.8)$ \\
$\quad$ upper sec. & $59.5(56.8-62.2)$ & $71.8(69.0-74.7)$ & $58.6(54.0-63.3)$ & $59.6(56.5-62.7)$ & $48.0(45.7-50.2)$ \\
tertiary & $66.9(64.1-69.7)$ & $77.7(74.7-80.6)$ & $66.8(62.0-71.7)$ & $67.9(64.7-71.1)$ & $54.2(51.9-56.5)$ \\
\hline
\end{tabular}




\begin{tabular}{|c|c|c|c|c|c|}
\hline Risk factor & Physical score & PF & RP & BP & $\mathrm{GH}$ \\
\hline $\begin{array}{l}\text { BMI } \\
\text { underweight } \\
\text { normal } \\
\text { overweight } \\
\text { obesity } \\
\end{array}$ & \begin{tabular}{|l|} 
a (a) \\
$78.7(61.9-95.6)$ \\
$71.0(67.4-74.6)$ \\
$63.9(60.5-67.3)$ \\
$55.7(52.8-58.6)$ \\
\end{tabular} & \begin{tabular}{|l} 
a (a) \\
$85.6(69.0-100.0)$ \\
$83.5(79.8-87.3)$ \\
$75.1(71.5-78.6)$ \\
$66.7(63.7-69.8)$ \\
\end{tabular} & $\begin{array}{l}\text { a (0.0763) } \\
81.2(52.1-100.0) \\
71.7(65.5-78.0) \\
64.3(58.5-70.2) \\
54.6(49.6-59.6) \\
\end{array}$ & $\begin{array}{l}\text { a (0.0005) } \\
88.3(69.9-100.0) \\
70.5(66.4-74.7) \\
64.4(60.4-68.3) \\
56.3(52.9-59.6) \\
\end{array}$ & $\begin{array}{l}\text { a (a) } \\
55.6(42.4-68.7) \\
57.7(54.8-60.7) \\
51.6(48.8-54.4) \\
44.9(42.5-47.3) \\
\end{array}$ \\
\hline $\begin{array}{c}\text { Smoking } \\
\text { never } \\
\text { former } \\
\text { current }\end{array}$ & \begin{tabular}{|l|}
$0.0118(0.0133)$ \\
$60.3(57.9-62.7)$ \\
$67.7(63.4-71.9)$ \\
$61.7(57.2-66.2)$ \\
\end{tabular} & \begin{tabular}{|l|}
$0.0027(0.0006)$ \\
$71.7(69.2-74.2)$ \\
$80.4(76.0-84.8)$ \\
$72.1(67.5-76.8)$ \\
\end{tabular} & $\begin{array}{l}0.0196(0.1631) \\
58.3(54.2-62.4) \\
69.9(62.7-77.0) \\
63.3(55.7-70.9) \\
\end{array}$ & $\begin{array}{l}0.1095(0.0529) \\
61.7(59.0-64.5) \\
67.0(62.2-71.9) \\
60.2(55.1-65.3) \\
\end{array}$ & $\begin{array}{l}0.0806(0.0939) \\
48.8(46.9-50.8) \\
53.3(49.8-56.8) \\
51.1(47.5-54.8) \\
\end{array}$ \\
\hline Risk factor & Mental score & VT & SF & RE & $\mathrm{MH}$ \\
\hline $\begin{array}{l}\text { Age } \\
\begin{array}{l}20-42 \\
43-55 \\
56-65 \\
66-90\end{array}\end{array}$ & \begin{tabular}{|l|}
$0.0005(0.0196)$ \\
$70.7(67.6-73.8)$ \\
$69.0(65.9-72.2)$ \\
$65.2(62.0-68.4)$ \\
$63.2(59.9-66.4)$ \\
\end{tabular} & $\begin{array}{l}0.0151(0.0301) \\
73.6(67.7-79.6) \\
72.4(66.3-78.5) \\
66.5(60.4-72.6) \\
63.3(57.0-69.6) \\
\end{array}$ & $\begin{array}{l}0.0011(0.0945) \\
54.4(51.4-57.3) \\
53.0(50.1-56.0) \\
50.8(47.8-53.8) \\
47.4(44.4-50.4) \\
\end{array}$ & \begin{tabular}{|l|}
$0.0123(0.1848)$ \\
$73.4(70.8-76.0)$ \\
$73.1(70.4-75.7)$ \\
$70.3(67.7-73.0)$ \\
$68.6(65.9-71.3)$ \\
\end{tabular} & \begin{tabular}{|l|}
$a(0.0016)$ \\
$81.1(77.4-84.9)$ \\
$77.7(73.8-81.5)$ \\
$72.5(68.7-76.3)$ \\
$70.2(66.4-74.1)$ \\
\end{tabular} \\
\hline $\begin{array}{c}\text { Gender } \\
\mathrm{F} \\
\mathrm{M} \\
\end{array}$ & \begin{tabular}{|l} 
a (a) \\
$64.0(61.9-66.1)$ \\
$71.4(69.0-73.8)$ \\
\end{tabular} & $\begin{array}{l}0.0057(0.0039) \\
65.4(61.4-69.4) \\
74.1(69.4-78.8) \\
\end{array}$ & $\begin{array}{l}\text { a (a) } \\
48.7(46.7-50.6) \\
55.2(53.0-57.5)\end{array}$ & \begin{tabular}{|l} 
a (a) \\
$68.4(66.7-70.1)$ \\
$75.5(73.5-77.6)$ \\
\end{tabular} & \begin{tabular}{|l|} 
a (a) \\
$71.7(69.2-74.2)$ \\
$80.7(77.7-83.6)$ \\
\end{tabular} \\
\hline $\begin{array}{l}\text { Education } \\
\text { basic } \\
\text { upper sec. } \\
\text { tertiary } \\
\end{array}$ & \begin{tabular}{|l|}
$0.0028(0.0097)$ \\
$62.9(57.3-68.6)$ \\
$65.0(62.7-67.3)$ \\
$70.2(67.8-72.6)$ \\
\end{tabular} & $\begin{array}{l}0.2138(0.3834) \\
67.3(56.4-78.1) \\
66.6(62.1-71.0) \\
72.2(67.6-76.8) \\
\end{array}$ & $\begin{array}{l}0.0012(0.0025) \\
45.3(40.0-50.6) \\
49.8(47.7-52.0) \\
54.3(52.0-56.5) \\
\end{array}$ & $\begin{array}{l}0.0004(0.0005) \\
68.1(63.4-72.9) \\
69.1(67.2-71.1) \\
74.4(72.4-76.4) \\
\end{array}$ & \begin{tabular}{|l|}
$0.0204(0.0316)$ \\
$71.1(64.2-77.9)$ \\
$73.4(70.6-76.2)$ \\
$78.4(75.6-81.3)$ \\
\end{tabular} \\
\hline $\begin{array}{l}\text { BMI } \\
\text { underweight } \\
\text { normal } \\
\text { overweight }\end{array}$ & \begin{tabular}{|l|}
$0.0067(0.2093)$ \\
$72.2(58.3-86.1)$ \\
$70.2(67.0-73.3)$ \\
$67.2(64.2-70.2)$ \\
\end{tabular} & $\begin{array}{l}0.3203(0.8980) \\
77.8(51.3-100.0) \\
73.5(67.5-79.5) \\
66.0(60.3-71.7) \\
\end{array}$ & $\begin{array}{l}0.0003(0.0177) \\
51.7(38.7-64.6) \\
54.1(51.2-57.0) \\
54.0(51.2-56.7) \\
\end{array}$ & \begin{tabular}{|l|}
$0.0030(0.0978)$ \\
$76.0(64.2-87.8)$ \\
$73.6(71.0-76.2)$ \\
$70.9(68.4-73.4)$ \\
\end{tabular} & \begin{tabular}{|l|}
$0.0043(0.3406)$ \\
$83.3(66.6-100.0)$ \\
$78.9(75.2-82.7)$ \\
$76.8(73.3-80.4)$ \\
\end{tabular} \\
\hline $\begin{array}{l}\text { Obesity } \\
\text { smoking } \\
\text { never } \\
\text { former } \\
\text { current }\end{array}$ & \begin{tabular}{|l|}
$65.4(62.8-67.9)$ \\
$0.0511(0.0731)$ \\
$67.2(65.1-69.2)$ \\
$70.1(66.5-73.6)$ \\
$63.6(59.8-67.4)$ \\
\end{tabular} & $\begin{array}{l}69.1(64.3-74.0) \\
0.0241(0.0233) \\
70.0(66.1-73.9) \\
74.0(67.2-80.8) \\
60.6(53.4-67.8)\end{array}$ & $\begin{array}{l}48.2(45.9-50.6) \\
0.0567(0.1700) \\
50.7(48.8-52.6) \\
55.0(51.7-58.3) \\
49.8(46.3-53.3)\end{array}$ & \begin{tabular}{|l|}
$69.8(67.7-72.0)$ \\
$0.1052(0.3574)$ \\
$71.3(69.6-73.0)$ \\
$73.8(70.9-76.8)$ \\
$69.2(66.0-72.3)$ \\
\end{tabular} & \begin{tabular}{|l|}
$72.6(69.5-75.6)$ \\
$0.5909(0.7319)$ \\
$74.9(72.5-77.4)$ \\
$77.5(73.1-81.8)$ \\
$74.9(70.3-79.5)$
\end{tabular} \\
\hline
\end{tabular}

HRQoL - health-related quality of life, PF - physical functioning, RP - role limitations due to physical problems, BP - body pain, GH - general health, VT - vitality, SF - social functioning, RE - role of limitations due to emotional problems, $\mathrm{MH}-$ mental health. Medians are given with a $95 \%$ confidence interval. $P$-values are given as non-adjusted and adjusted values (in brackets), with a $=p<0.0001$.

\begin{tabular}{|c|c|c|c|c|c|c|c|c|c|c|c|c|c|c|c|}
\hline \multirow{2}{*}{$\begin{array}{l}\text { CKD low } \\
\text { group } \\
\text { Physical } \\
\text { score }\end{array}$} & \multicolumn{3}{|c|}{ Sitting $p$} & \multicolumn{3}{|c|}{ MET walking $p$} & \multicolumn{3}{|c|}{ MET moderate $p$} & \multicolumn{3}{|c|}{ MET vigorous $p$} & \multicolumn{3}{|c|}{ TOTAL MET $p$} \\
\hline & -0.04 & $(0.7497)$ & {$[1.0]$} & 0.45 & $(0.0002)$ & {$[0.005]$} & 0.12 & $(0.2960)$ & {$[1.0]$} & 0.28 & $(0.0177)$ & [0.30] & 0.39 & $(0.0014)$ & {$[0.032]$} \\
\hline $\mathrm{PF}$ & -0.08 & $(0.5669)$ & {$[1.0]$} & 0.44 & $(0.0003)$ & {$[0.006]$} & 0.19 & $(0.1171)$ & {$[1.0]$} & 0.29 & $(0.0146)$ & {$[0.26]$} & 0.37 & $(0.0022)$ & [0.045] \\
\hline $\mathrm{RP}$ & -0.06 & $(0.6782)$ & {$[1.0]$} & 0.44 & $(0.0003)$ & {$[0.006]$} & 0.05 & $(0.6848)$ & [1.0] & 0.28 & $(0.0187)$ & [0.30] & 0.33 & $(0.0071)$ & [0.142] \\
\hline $\mathrm{BP}$ & 0.20 & $(0.1433)$ & {$[1.0]$} & 0.31 & $(0.0102)$ & {$[0.194]$} & -0.07 & (0.5535) & [1.0] & 0.17 & $(0.1586)$ & [1.0] & 0.21 & $(0.0771)$ & [1.0] \\
\hline GH & -0.17 & $(0.2100)$ & [1.0] & 0.23 & $(0.0520)$ & {$[0.728]$} & 0.21 & $(0.0772)$ & {$[1.0]$} & 0.09 & $(0.4645)$ & [1.0] & 0.27 & $(0.0266)$ & {$[0.4]$} \\
\hline $\begin{array}{l}\text { Mental } \\
\text { score }\end{array}$ & 0.03 & $(0.8135)$ & [1.0] & 0.33 & $(0.0065)$ & {$[0.2]$} & 0.16 & $(0.1846)$ & [1.0] & 0.22 & $(0.0634)$ & {$[1.0]$} & 0.27 & $(0.0227)$ & {$[0.5]$} \\
\hline VT & 0.12 & $(0.3680)$ & {$[1.0]$} & 0.28 & $(0.0215)$ & {$[0.5]$} & 0.06 & $(0.5884)$ & {$[1.0]$} & 0.13 & $(0.2905)$ & [1.0] & 0.17 & $(0.1559)$ & [1.0] \\
\hline SF & 0.02 & $(0.8694)$ & {$[1.0]$} & 0.19 & $(0.1120)$ & {$[1.0]$} & 0.16 & $(0.1751)$ & {$[1.0]$} & 0.22 & $(0.0631)$ & {$[1.0]$} & 0.22 & $(0.0624)$ & [1.0] \\
\hline $\mathrm{RE}$ & 0.00 & $(0.9725)$ & [1.0] & 0.27 & $(0.0274)$ & [0.6] & 0.16 & $(0.1826)$ & [1.0] & 0.08 & $(0.5001)$ & {$[1.0]$} & 0.23 & $(0.0549)$ & [1.0] \\
\hline $\mathrm{MH}$ & -0.03 & $(0.8382)$ & [1.0] & 0.23 & $(0.0543)$ & [1.0] & 0.07 & $(0.5531)$ & [1.0] & 0.21 & $(0.0834)$ & [1.0] & 0.21 & $(0.0869)$ & [1.0] \\
\hline
\end{tabular}

\begin{tabular}{|l|l|l|l|l|l|l|l|l|l|l|l|l|l|l|l|l|}
\hline $\begin{array}{l}\text { CKD high } \\
\text { group }\end{array}$ & \multicolumn{3}{|l|}{ Sitting $p$} & \multicolumn{3}{l|}{ MET walking $p$} & \multicolumn{3}{l|}{ MET vigorous $p$} & \multicolumn{2}{l|}{ TOTAL MET $p$} \\
\hline $\begin{array}{l}\text { Physical } \\
\text { score }\end{array}$ & 0.07 & $(0.6971)$ & {$[1.0]$} & -0.05 & $(0.7228)$ & {$[1.0]$} & 0.12 & $(0.3983)$ & {$[1.0]$} & 0.17 & $(0.2407)$ & {$[1.0]$} & 0.09 & $(0.5676)$ & {$[1.0]$} \\
\hline PF & 0.06 & $(0.7436)$ & {$[1.0]$} & 0.02 & $(0.8857)$ & {$[1.0]$} & 0.25 & $(0.0858)$ & {$[1.0]$} & 0.24 & $(0.1061)$ & {$[1.0]$} & 0.19 & $(0.2029)$ & {$[1.0]$} \\
\hline RP & 0.01 & $(0.9371)$ & {$[1.0]$} & -0.03 & $(0.8242)$ & {$[1.0]$} & 0.06 & $(0.6722)$ & {$[1.0]$} & 0.08 & $(0.5987)$ & {$[1.0]$} & 0.07 & $(0.6261)$ & {$[1.0]$} \\
\hline BP & 0.16 & $(0.3646)$ & {$[1.0]$} & -0.22 & $(0.1431)$ & {$[1.0]$} & -0.04 & $(0.7642)$ & {$[1.0]$} & 0.01 & $(0.9268)$ & {$[1.0]$} & -0.18 & $(0.2233)$ & {$[1.0]$} \\
\hline GH & -0.15 & $(0.3982$ & {$[1.0]$} & 0.13 & $(0.3683)$ & {$[1.0]$} & 0.31 & $(0.0345)$ & {$[0.83]$} & 0.25 & $(0.0866)$ & {$[1.0]$} & 0.36 & $(0.0159)$ & {$[0.4]$} \\
\hline
\end{tabular}




\begin{tabular}{|l|l|l|l|l|l|l|l|l|l|l|l|l|l|l|l|l|}
\hline $\begin{array}{l}\text { CKD high } \\
\text { group }\end{array}$ & \multicolumn{3}{|l|}{ Sitting $p$} & \multicolumn{3}{l|}{ MET walking $p$} & \multicolumn{3}{l|}{ MET moderate $p$} & \multicolumn{3}{l|}{ MET vigorous $p$} & \multicolumn{3}{l|}{ TOTAL MET $p$} \\
\hline $\begin{array}{l}\text { Mental } \\
\text { score }\end{array}$ & 0.03 & $(0.8812)$ & {$[1.0]$} & 0.02 & $(0.8795)$ & {$[1.0]$} & 0.05 & $(0.7489)$ & {$[1.0]$} & 0.08 & $(0.5676)$ & {$[1.0]$} & 0.07 & $(0.6586)$ & {$[1.0]$} \\
\hline VT & 0.26 & $(0.1369)$ & {$[1.0]$} & 0.01 & $(0.9680)$ & {$[1.0]$} & -0.17 & $(0.2593)$ & {$[1.0]$} & -0.15 & $(0.2952)$ & {$[1.0]$} & -0.12 & $(0.4272)$ & {$[1.0]$} \\
\hline SF & -0.10 & $(0.5636)$ & {$[1.0]$} & 0.06 & $(0.6680)$ & {$[1.0]$} & 0.26 & $(0.0750)$ & {$[1.0]$} & 0.08 & $(0.5785)$ & {$[1.0]$} & 0.20 & $(0.1819)$ & {$[1.0]$} \\
\hline RE & 0.02 & $(0.8901)$ & {$[1.0]$} & 0.17 & $(0.2449)$ & {$[1.0]$} & 0.12 & $(0.3971)$ & {$[1.0]$} & 0.24 & $(0.0981)$ & {$[1.0]$} & 0.24 & $(0.1092)$ & {$[1.0]$} \\
\hline MH & -0.05 & $(0.7913)$ & {$[1.0]$} & 0.10 & $(0.5146)$ & {$[1.0]$} & 0.15 & $(0.2936)$ & {$[1.0]$} & 0.16 & $(0.2628)$ & {$[1.0]$} & 0.15 & $(0.3240)$ & {$[1.0]$} \\
\hline
\end{tabular}

HRQoL - health-related quality of life, PA - physical activity, CKD - chronic kidney disease, MET - metabolic unit, PF - physical functioning, RP - role limitations due to physical problems, BP - body pain, GH - general health, VT - vitality, SF - social functioning, RE - role of limitations due to emotional problems, $\mathrm{MH}-$ mental health.

\section{Discussion}

There is consistent evidence that CKD patients benefit from physical activity $[2,25]$. The main finding of our study is that concerning the associations between lifestyle-related factors and HRQoL, we found that CKD patients with moderate-severe kidney function loss (GFR $<45 \mathrm{~mL} / \mathrm{min} / 1.7 \mathrm{~m}^{2}$ ), who reported about good walking habits, have higher quality of life scores as well. Kosmadakis et al. have shown that regular walking has several positive outcomes in CKD patients [11], and a recent study had demonstrated that physical function and walking are even linked to survival in CKD patients [18].

We demonstrated that CKD patients with markedly lowered kidney function value their mental and physical HRQoL in all subscales of physical and mental scorings, except RE, which was significantly lower in comparison to patients with higher eGFR and with CC group patients. We showed decreasing trends of HRQoL in different stages of CKD similarly to the results of $\mathrm{Ag}$ garwal et al. study [26].

It is a well-known fact that light PA is beneficial for CKD patients to prevent CV events. Unfortunately, similarly to our results, many authors have demonstrated that most individuals living with CKD have low PA and probably need more resources to support and maintain a physically active lifestyle [27].

Self-reported IPAQ may underestimate light PA, which is the main form of PA in the CKD population. Rosa et al. have shown that IPAQ may underestimate light PA [28]. However, the high reliability of IPAQ questionnaire has been found in assessing walking habits [29].

Factors contributing to poor utilisation of renal rehabilitation should be individually determined, and exercise therapy should be considered as an important therapeutic modality for the comprehensive management of CKD patients. The importance of regular PA in lifestyle changes in patients with CKD should not be overlooked. Walking habits could be fostered in such ways that they become a routine part of the lifestyle of CKD patients early in the treatment and supervision period and in the mind-set of patients who then strive for self-improvement. It requires much effort to encourage patients to increase the level of PA by combining tasks of everyday life with more walking, using stairs instead of an elevator, leaving the car far away from the place of work, practicing ergonomic gardening, load moving and lifting techniques, etc. Patients often think that only exercise with special equipment and supervision can be con- sidered physical activity, and they pay less attention to how to combine healthy exercising with their activities, especially when considering how to increase everyday walking habits. Effective cooperation between general physicians, renal nurses and nephrologists is essential to ensure a better consulting system for CKD patients [30].

\section{Limitations of the study}

We are aware that questionnaires deliver subjective insights of HRQoL and PA. The choice of questionnaires we used in the study were based on knowledge that both IPAQ and SF-36 are widely used throughout the world. The cross-sectional study can provide only obscure implications of relationships between lifestyle and HRQoL in patients with CKD. HRQoL depends on many factors, including modality of treatment, which was not assessed in the study. However, the gathered information improves our knowledge about the HRQoL and PA of CKD patients.

\section{Conclusions}

The main aim of the study was to find associations between PA and self-reported patient outcomes in CKD patients, and we found that among the subjects with severely impaired kidney function, walking habits plays an important role in HRQoL. CKD patients with eGFR $<45 \mathrm{~mL} / \mathrm{min} / 1.73 \mathrm{~m}^{2}$, who reported about good walking habits, had higher quality of life scores.

The importance of regular PA in patients with CKD should not be overlooked. Walking habits could be fostered in such a way that it becomes a routine part of the lifestyle of CKD patients to prevent cardiovascular complications and improve prognosis and quality of life.

Tests for physical activity and quality of life measurement are routinely used by general practitioners, and this is a good way toward personalised medicine for better control of CKD patients.

Acknowledgements. For the current study, data was collected under the two large institutional projects: "Alcohol and nutrition as the predictors of progress and outcome of chronic conditions" and "Connective tissue and vascular injury in chronic disease". The study was financially supported by scientific grant IUT2-8. The authors thank the doctors Mart Kull, Kati Kärberg, Anni Tolk and Annika Valner for their kind help in data collection.

Source of funding: This work was funded from the authors' own resources. Conflicts of interest: The authors declare no conflicts of interest.

\section{References}

1. Ridao N, Luno J, Garcia de Vinuesa S, et al. Prevalence of hypertension in renal disease. Nephrol Dial Transplant 2001; 16(Suppl. 1): 70-73.

2. Painter $P$, Roshanravan B. The association of physical activity and physical function with clinical outcomes in adults with chronic kidney disease. Curr Opin Nephrol Hypertens 2013; 22(6): 615-623. 
3. Craenenbroeck AH van, Craenenbroeck EM van, Ackeren $\mathrm{K}$ van, et al. Impaired vascular function contributes to exercise intolerance in chronic kidney disease. Nephrol Dial Transplant 2016; 31(12): 2064-2072.

4. Orth SR. Smoking - a risk factor for progression of renal disease. Kidney Blood Press Res 2000; 23(3-5): 202-204.

5. Xia J, Wang L, Ma Z, et al. Cigarette smoking and chronic kidney disease in the general population: a systematic review and metaanalysis of prospective cohort studies. Nephrol Dial Transplant 2017; 32(3), doi: 10.1093/ndt/gfw452.

6. Ritz E, Schwenger V. Lifestyle modification and progressive renal failure. Nephrology (Carlton) 2005; 10(4): 387-392.

7. Foley RN, Parfrey PS, Sarnak MJ. Clinical epidemiology of cardiovascular disease in chronic renal disease. Am J Kidney Dis 1998; 32(5, Suppl. 3): S112-S119.

8. Luft FC. Renal disease as a risk factor for cardiovascular disease. Basic Res Cardiol 2000; 95(Suppl. 1): 172-176.

9. Sarnak MJ, Coronado BE, Greene T, et al. Cardiovascular disease risk factors in chronic renal insufficiency. Clin Nephrol 2002; 57(5): 327-335.

10. KDIGO. KDIGO 2012 Clinical practice guideline for the evaluation and management of chronic kidney disease. Kidney Int 2013; 3(1): 1-163.

11. Kosmadakis GC, John SG, Clapp EL, et al. Benefits of regular walking exercise in advanced pre-dialysis chronic kidney disease. Nephrol Dial Transplant 2012; 27(3): 997-1004.

12. Navaneethan SD, Yehnert H, Moustarah F, et al. Weight loss interventions in chronic kidney disease: a systematic review and metaanalysis. Clin J Am Soc Nephrol 2009; 4(10): 1565-1574.

13. Pei G, Tang Y, Tan L, et al. Aerobic exercise in adults with chronic kidney disease (CKD): a meta-analysis. Int Urol Nephrol 2019; 51(10): 1787-1795.

14. Villanego F, Naranjo J, Vigara L, et al. Impact of physical exercise in patients with chronic kidney disease: systematic review and metaanalysis. Nefrologia 2020; 40(3): 237-252.

15. Beto JA, Bansal VK. Medical nutrition therapy in chronic kidney failure: integrating clinical practice guidelines. J Am Diet Assoc 2004; 104 (3): 404-409.

16. Heiwe S, Jacobson SH. Exercise training for adults with chronic kidney disease. Cochrane Database Syst Rev 2011; 10: CD003236.

17. Gansevoort RT, Correa-Rotter R, Hemmelgarn BR, et al. Chronic kidney disease and cardiovascular risk: epidemiology, mechanisms, and prevention. Lancet 2013; 382(9889): 339-352.

18. MacKinnon HJ, Wilkinson TJ, Clarke AL, et al. The association of physical function and physical activity with all-cause mortality and adverse clinical outcomes in nondialysis chronic kidney disease: a systematic review. Ther Adv Chronic Dis 2018; 9(11): $209-226$.

19. Tripepi G, D'Arrigo G, Jager KJ, et al. Do we still need cross-sectional studies in Nephrology? Yes we do! Nephrol Dial Transplant 2017; 32(Suppl. 2): ii19-ii22, doi: 10.1093/ndt/gfw439.

20. Polluste K, Aart A, Kallikorm R, et al. Adverse lifestyle and health-related quality of life: gender differences in patients with and without chronic conditions. Scand J Public Health 2016; 44(2): 209-216.

21. Craig CL, Marshall AL, Sjostrom M, et al. International physical activity questionnaire: 12 -country reliability and validity. Med Sci Sports Exerc 2003; 35(8): 1381-1395.

22. Painter P, Marcus RL. Assessing physical function and physical activity in patients with CKD. Clin J Am Soc Nephrol $2013 ; 8(5): 861-872$.

23. Lowrie EG, Curtin RB, LePain N, et al. Medical outcomes study short form-36: a consistent and powerful predictor of morbidity and mortality in dialysis patients. Am J Kidney Dis 2003; 41(6): 1286-1292.

24. Wang HM, Beyer M, Gensichen J, et al. Health-related quality of life among general practice patients with differing chronic diseases in Germany: cross sectional survey. BMC Public Health 2008; 8: 246.

25. Pechter U, Raag M, Ots-Rosenberg M. Regular aquatic exercise for chronic kidney disease patients: a 10-year follow-up study. Int J Rehabil Res 2014; 37(3): 251-255.

26. Aggarwal HK, Jain D, Pawar S, et al. Health-related quality of life in different stages of chronic kidney disease. QJM 2016; 109(11): 711-716.

27. Parsons TL, Bohm C, Poser K. "A learned soul to guide me": The voices of those living with kidney disease inform physical activity programming. Physiother Can 2018; 70(3): 289-295.

28. Rosa CS, Gracia-Marco L, Barker AR, et al. Assessment of physical activity by accelerometer and IPAQ-Short version in patients with chronic kidney disease undergoing hemodialysis. Blood Purif 2015; 40(3): 250-255.

29. Ploeg HP van der, Tudor-Locke C, Marshall AL, et al. Reliability and validity of the international physical activity questionnaire for assessing walking. Res Q Exerc Sport 2010; 81(1): 97-101.

30. Starkopf A, Müller R, Starkopf A, et al. Physical function measures and health-related quality of life in primary care medicine: crosssectional study. Fam Med Prim Care Rev 2017; 19(2): 161-166.

Tables: 3

Figures: 1

References: 30

Received: 23.03.2020

Reviewed: 9.04.2020

Accepted: 11.05 .2020

Address for correspondence:

Jana Uhlinova, MD

Department of Internal Medicine

University of Tartu

L. Puusepa 8

Tartu 50406

Estonia

Tel.: +372 5544495

E-mail: jana.uhlinova@kliinikum.ee 